\title{
Graphene Oxides Derivatives Prepared by an Electrochemical Approach ${ }^{\dagger}$
}

\author{
Carlos Sainz-Urruela ${ }^{1}$, Soledad Vera-López ${ }^{1,2}$, María Paz San Andrés ${ }^{1,2}$ and \\ Ana M. Díez-Pascual 1,2,* \\ 1 Department of Analytical Chemistry, Physical Chemistry and Chemical Engineering, Faculty of Sciences, \\ University of Alcalá, 28805 Alcalá de Henares, Madrid, Spain \\ 2 Institute of Chemistry Research, “Andrés M. del Río” (IQAR), University of Alcalá, \\ Ctra. Madrid- Barcelona Km. 33.6, 28805 Alcalá de Henares, Madrid, Spain \\ * Correspondence: am.diez@uah.es; Tel.: +34-918-856-430 \\ + Presented at the 2nd International Online-Conference on Nanomaterials, 15-30 November 2020; Available \\ online: https://iocn2020.sciforum.net/.
}

Published: 15 November 2020

\begin{abstract}
Graphene oxide (GO) is a novel material that can be defined as a single monolayer of graphite with oxygen-containing functionalities such as epoxides, alcohols, and carboxylic acids. It is an interesting alternative to graphene for many applications due to its exceptional optical, chemical, and electrical properties. In this study, GOs with different extent of surface groups were prepared by an electrochemical two-stage approach using graphite as raw material. Various synthesis conditions were tested to increase the nanomaterial oxidation level, and the surface topography of the GO derivatives was analyzed via Scanning Electron Microscopy (SEM) and atomic force microscopy (AFM). The electrochemical approach employed in this study maintains the integrity of the graphene sheets, allowing to get large, uniform and well exfoliated GO. A correlation was found between the derivatives properties and their surface topography, interlayer spacing, defect content and specific surface area (SSA). In particular, the electrical resistance decreases with increasing SSA while rises with increasing the $D / G$ band intensity ratio in the Raman spectra, hence the defect content. Understanding the structure-property relationships in these materials is useful for the design of modified GOs with controlable morphologies and properties for a wide range of applications in electrical/electronic devices.
\end{abstract}

Keywords: graphene oxide; oxidation level; structure-property relationship; surface topography; specific surface area; interlayer spacing

\section{Introduction}

Lately, considerable interest has been directed to graphene and its derivatives because of their unique properties and broad range of applications in electrical/electronic and biomedical devices. This oxidized form of graphene with several surface oxygen-containing groups such as epoxides, alcohols, and carboxylic acids is currently been used as batteries, electrical cells, nanocomposites, besides to gain importance in biomedicine [1-6]. The greatest interest in GO over G could be explained by its higher possibilities to achieve functional properties since it is feasible to tailor its functionalities through reduction and functionalisation processes $[7,8]$. Further, it is possible to prepare novel thin films and flexible composites at a cheaper cost compared to other carbon-based nanomaterials $[9,10]$. These materials can be used as fillers in polymer matrices due to their $2 \mathrm{D}$ lamellar structure, very high specific surface area (SSA) and their nature to disperse in a wide range of organic and inorganic solvents [11-14]. 
The control of the oxidation level can be useful for the design of GOs with tailorable structural, electrical, mechanical, thermal and optical properties. For instance, GO is typically electrical insulating, albeit by controlling the synthesis conditions, its conductivity can be improved and conducting or semiconducting products, such as composites and thin films can be developed [15-17]. The experimental conditions set for the synthesis process are essential, since they modify a number of physical properties including inter layer distance, SSA, defect content, and so forth. Hence, derivatives from an application viewpoint, it is crucial to understand how the change in those conditions affects the properties of GO-based nanomaterials [18]. To the best of our knowledge, very few studies have focused on investigating the correlation between SSA, interlayer spacing and defect content with the mechanical, physical and electrical properties of such nanomaterials [19]. In particular, SSA can directly influence the electrical properties, hence obtaining a large SSA in graphene materials is highly desirable. On the other hand, the GO band gap rises as the C/O ratio diminishes [20], which offers an effective approach to tailor the optical properties of these nanomaterials. Besides, as the oxidation level increases, both the Young's modulus and tensile strength are expected to drop gradually owed to the breakage of the $\mathrm{sp}^{2}$ carbon network and reduction of the energetic stability for the ordered GO [21]. Nonetheless, the rise in the GO oxidation level is beneficial for enhancing the mechanical properties of nanocomposites [13], particularly for polymeric matrices with oxygenated groups such as chitosan [22] or polyamide [23]. The heat capacity, thermal conductivity and specific capacitance can also be modified via tailoring the level of oxidation [24,25].

The synthesis methods for GO can be divided into two major categories: (a) bottom-up approaches (such as chemical vapor deposition, epitaxial growth on silicon carbide wafers, etc.) in which carbon molecules are used to make raw graphene; these have been shown to be timeconsuming and difficult to scale-up [26]. (b) top-down methods in which layers of graphene derivatives are obtained from a carbon source, usually graphite; these are more popular, principally for use in nanocomposite materials. The first GO synthesis is attributed to Brodie, [27] Staudenmaier, and Hummers and Offeman [28], who obtained graphite oxide via the oxidation of graphite through various techniques. Any method that makes changes to the synthesis pathway proposed by Hummers can be regarded as a "modified Hummers method" [29].

Lately, electrochemical processes have arisen as environmentally friendly and versatile alternatives to synthesize carbon nanomaterials due to of their efficiency and low cost [30]. Previous works have produced GO via electrochemical oxidation of different graphitic materials including graphite powders, rods, foils, plates or even pencil cores [31-35]. Nonetheless, the electrolysis procedure usually deteriorates the delamination of the graphitic materials, yielding products with poor exfoliation levels, and result in low-yield production [36].

In a previous study [37], we reported the synthesis of electrochemically exfoliated graphene oxides (EGOs) with different amount of surface groups. The EGOs preparation process comprised two steps: firstly a mild intercalation stage of $\mathrm{SO}_{4^{2-}}$ ions within the graphite sheets, resulting in a graphite intercalation compound (GIC) and then an oxidation/exfoliation stage of the GIC under stronger conditions. The results were compared to those obtained for a reference GO synthesized via a modified Hummers' method. The aim of the current work is to correlate the surface topography, interlayer spacing, defect content and specific surface area (SSA) of the synthesized EGOs with their macroscopic properties.

\section{Materials and Methods}

\subsection{Materials and Reagents}

Flexible graphite foil (FGF, $\mathrm{d}_{25^{\circ} \mathrm{C}}=1.00 \mathrm{~g} / \mathrm{cm}^{3}, \mathrm{C}: 99.5 \%, \mathrm{~S}<300 \mathrm{ppm}, \mathrm{Cl}<50 \mathrm{ppm}$, ash $<1 \%$, thickness $0.1 \mathrm{~mm}$ ) was supplied by Beyond Materials, Inc. (Tucson, AZ, USA) and dried in an oven at $60{ }^{\circ} \mathrm{C}$ for 48 $\mathrm{h}$ before use. Powdered graphite flakes (SP-1, $\mathrm{d}_{25} 5^{\circ} \mathrm{C}=1.05 \mathrm{~g} / \mathrm{cm}^{3}, \mathrm{C}: 99.9 \%$, ash $<0.5 \%$, average size 30-150 $\mu \mathrm{m})$ were purchased from Bay Carbon, Inc. (Bay City, Michigan, USA) and dried under identical conditions. $\mathrm{KMnO}_{4}, \mathrm{H}_{2} \mathrm{SO}_{4}, \mathrm{~K}_{2} \mathrm{~S}_{2} \mathrm{O}_{8}, \mathrm{P}_{2} \mathrm{O}_{5}, \mathrm{H}_{2} \mathrm{O}_{2}$ (30 wt $\%$ in water) and platinum wire, (ø: $0.5 \mathrm{~mm}, 99.99 \%$ trace metals basis) were obtained from Sigma-Aldrich and used as received. Ultrapure water was purified by a Millipore Elix 15824 Advantage 15 UV system (Millipore, Burlington, MA, USA). 


\subsection{Preparation of GOs via a Two-Stage Electrochemical Process}

Electrochemically exfoliated graphene oxides (EGOs) were prepared from FGF through an electrochemical procedure that included two stages: the first was performed in an electrolysis cell with a slice of FGF fixed onto a tungsten wire by silver glue as anode, a Pt wire as cathode and 98 $w \mathrm{t} \% \mathrm{H}_{2} \mathrm{SO}_{4}$ diluted in $100 \mathrm{~mL}$ of Milli-Q water as an electrolyte. A voltage of 1 or $2 \mathrm{~V}$ was initially applied for 10 or $30 \mathrm{~min}$, leading to formation of a graphite intercalation compound (GIC). The second stage consisted in the electrochemical oxidation of the GIC that acted as anode, a Pt wire as cathode and 40,65 or $98 \mathrm{wt} \% \mathrm{H}_{2} \mathrm{SO}_{4}$ as an electrolyte. A high voltage (10-30 V) was applied for periods between 30 and $120 \mathrm{~s}$. A more detailed description of the synthesis procedure is given in [37].

Table 1. Nomenclature and experimental conditions for the different EGO samples.

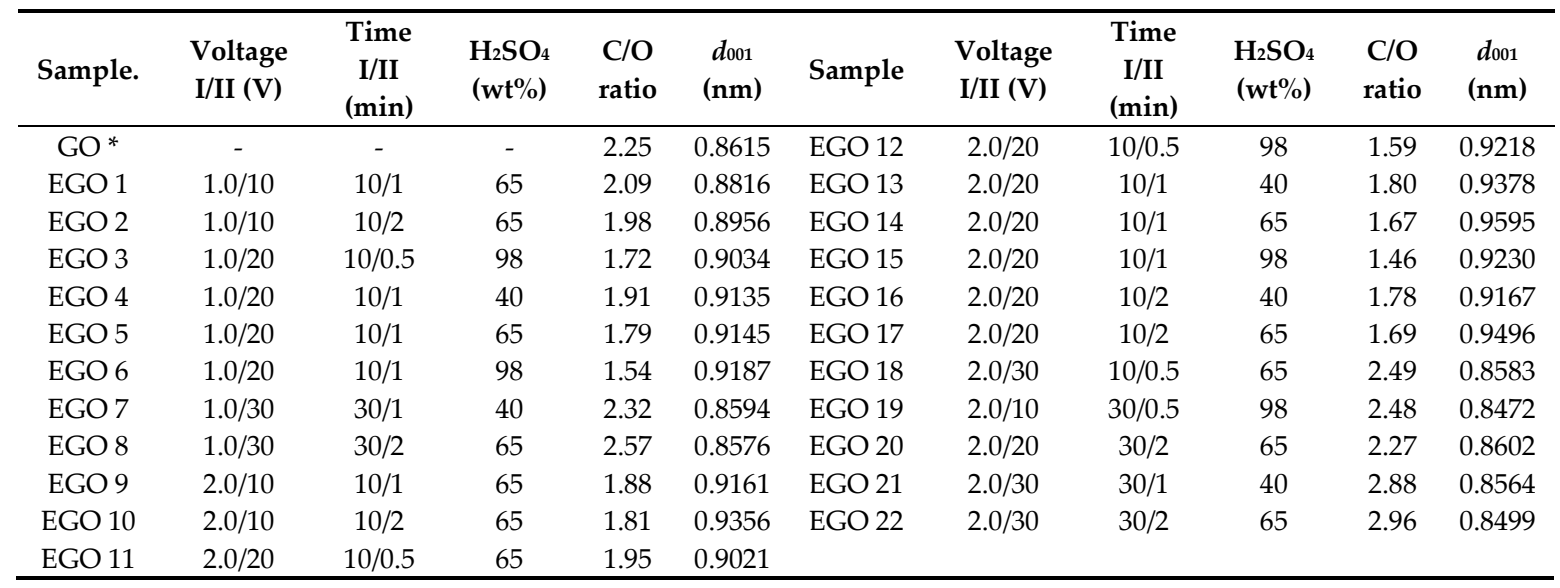

* Synthesized by a modified Hummers' method. I and II refer to the intercalation and exfoliation stages, respectively. The $\mathrm{C} / \mathrm{O}$ ratio has been calculated from elemental analysis measurements, and $d$ is the interlayer spacing corresponding to the (001) reflection of GO.

\subsection{Characterization}

A LECO CHNS-932 elemental analyzer was used to perform elemental analysis measurements.

The morphology was analyzed with a SU8000 Hitachi scanning electron microscope (SEM), operating at $15.0 \mathrm{kV}$ and an emission current of $10 \mathrm{~mA}$.

Atomic force microscopy (AFM) imaging was performed using a Bruker Dimension Icon system coupled with a Nanoscope V controller, using Peakforce QNM imaging mode and a $100 \mu \mathrm{m}$ long monolithic silicon cantilever.

X-ray diffraction (XRD) measurements were carried out on a Bruker D8 Advance diffractometer fitted with a $\mathrm{Cu}$ X-ray tube and a $\mathrm{Ni}_{\beta}$ filter operating at $40 \mathrm{kV}$ and an intensity of $40 \mathrm{~mA}$.

Raman spectra were acquired at room temperature with a laser output power of $1 \mathrm{~mW}$ using a Renishaw Raman microscope incorporating a He-Ne laser $(632.8 \mathrm{~nm})$. To minimize the signal-tonoise ratio, at least 10 scans were recorded for each sample. Data were then processed with the WiRE 3.3 Renishaw software, and the spectra were normalized to the $G$ band for the sake of comparison.

Brunauer-Emmett-Teller (BET) specific surface area analysis was performed via nitrogen adsorption-desorption measurements at $77 \mathrm{~K}$ with a Quantachrom Autosorb IQ-C gas adsorption system. Prior to the analysis, moisture content was removed by drying for 2 days at $80^{\circ} \mathrm{C}$.

The electrical resistivity of the synthesized EGOs was determined at room temperature under a pressure of $600 \mathrm{kPa}$ set by using an upper weight, with a KEITHLEY 2182A nanovoltmeter and a KEITHLEY 6221 current source, respectively. Prior to the measurements, each sample was positioned in a Teflon cylinder and compressed for $1 \mathrm{~h}$ between two stainless steel plates that acted as electrodes. $R_{s}$ was calculated as: $R_{s}=4532 \times(V / I)$, being $V$ the test voltage and I the current. 


\section{Results and Discussion}

\subsection{Topography of the Synthesized EGOs}

The surface topography of FGF and the synthesized EGOs was examined by SEM, and typical images of the reference sample synthesized by the Hummers' method (GO*), EGO 2, EGO 5, EGO 14, EGO 15 and EGO 21 are compared in Figure 1.
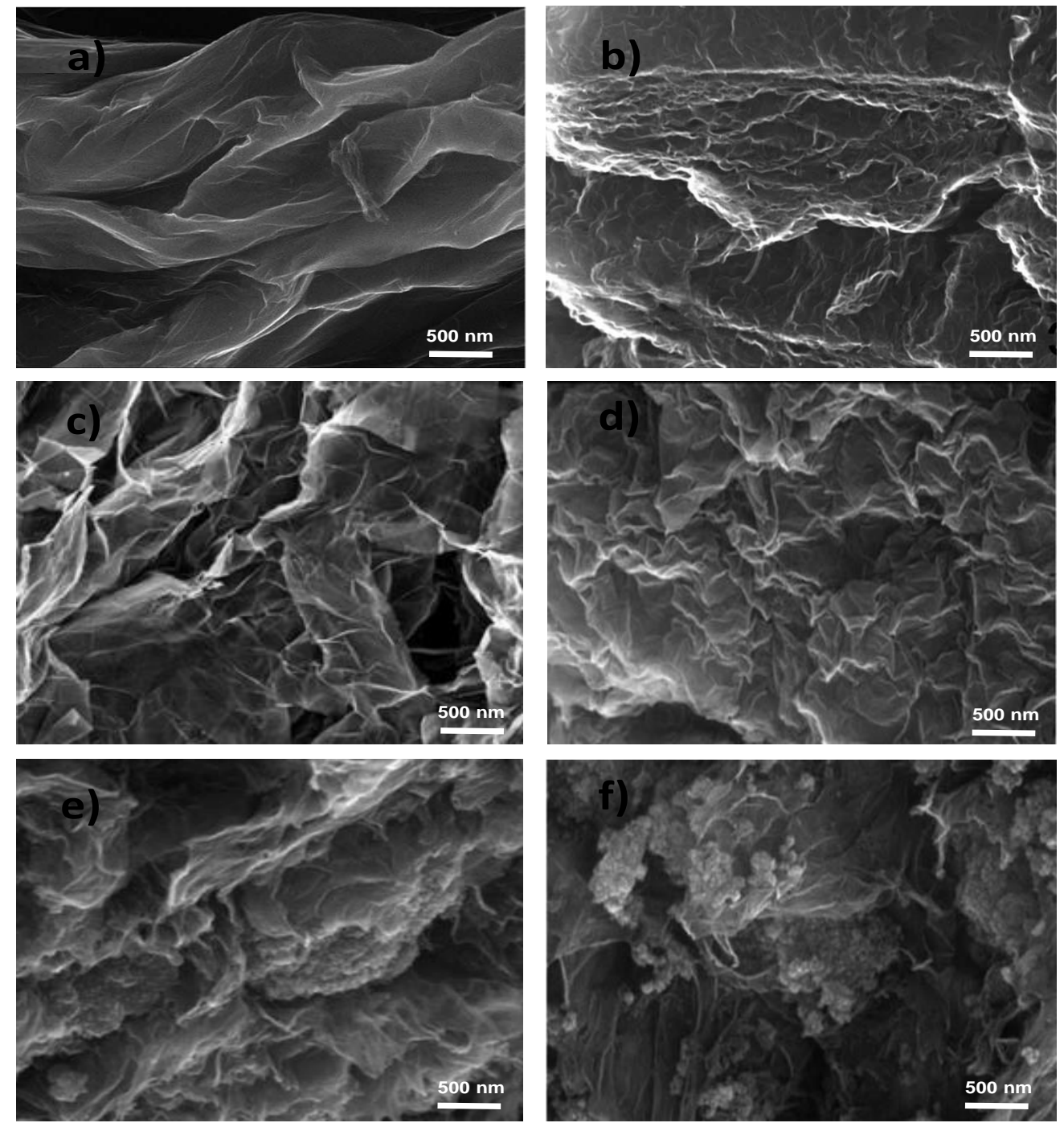

Figure 1. Representative SEM images of EGO 14 (a), EGO 15 (b), EGO 5 (c), reference GO* (d), EGO 2 (e) and EGO 21 (f).

The image of EGO 14 (Figure 1a), obtained by applying a low bias of $2 \mathrm{~V}$ for $10 \mathrm{~min}$ followed by oxidation under $20 \mathrm{~V}$ for $60 \mathrm{~s}$ using $65 \% \mathrm{H}_{2} \mathrm{SO}_{4}$ as electrolyte, reveals a rough surface topography with well-exfoliated and very well detached graphene sheets, with thicknesses in the range of 5-15 $\mathrm{nm}$. Analogous curvatures and surface folds have been previously reported for GO functionalized with amines [38], ascribed to increased number of regular hydrogen bonds between the amine moieties. Thus, EGO 14 presents a low C/O ratio (Table 1), that is, a high oxidation level, likely with a very high content of carboxylic acid groups [37], preferentially located at the sheet edges, which are able to interact via hydrogen bonding.

A good exfoliation level is also found for EGO 15 (Figure 1b), in which the electrolyte concentration was increased to $98 \%$ and the rest of conditions were maintained (Table 1), hence it exhibits higher oxidation level. On the other hand, a less efficient exfoliation and oxidation, albeit with considerably folding, is found for EGO 5 (Figure 1c), in which the low voltage was decreased to 
$1 \mathrm{~V}$ and the other conditions were similar to those of EGO 14. A denser and more compact structure is also observed for the reference GO synthesized by the Hummer's method (Figure 1d), which shows a C/O ratio of 2.25, and layer thicknesses up to $30 \mathrm{~nm}$ in good agreement with previous works [26].

On the other hand, the image of EGO 2 (Figure 1e) reveals a mild exfoliation, with connected flakes forming a compact network, likely related to the fact that the low voltage was applied for a long time. Regarding EGO 21 (Figure 1f), in which the voltage of the second stage is higher than 20 $\mathrm{V}$, large GO aggregates can be observed, since the exfoliation rate should be very fast, also resulting in thick flakes (i.e., $\geq 20 \mathrm{~nm}$ ). Likely, only the lateral parts of the sheets are oxidized, thus resulting in a very high $\mathrm{C} / \mathrm{O}$ ratio (low oxidation degree).

Further information about the surface topography of the EGOs was obtained from AFM, and representative images of EGO 14 and EGO 21 are compared in Figure 2.
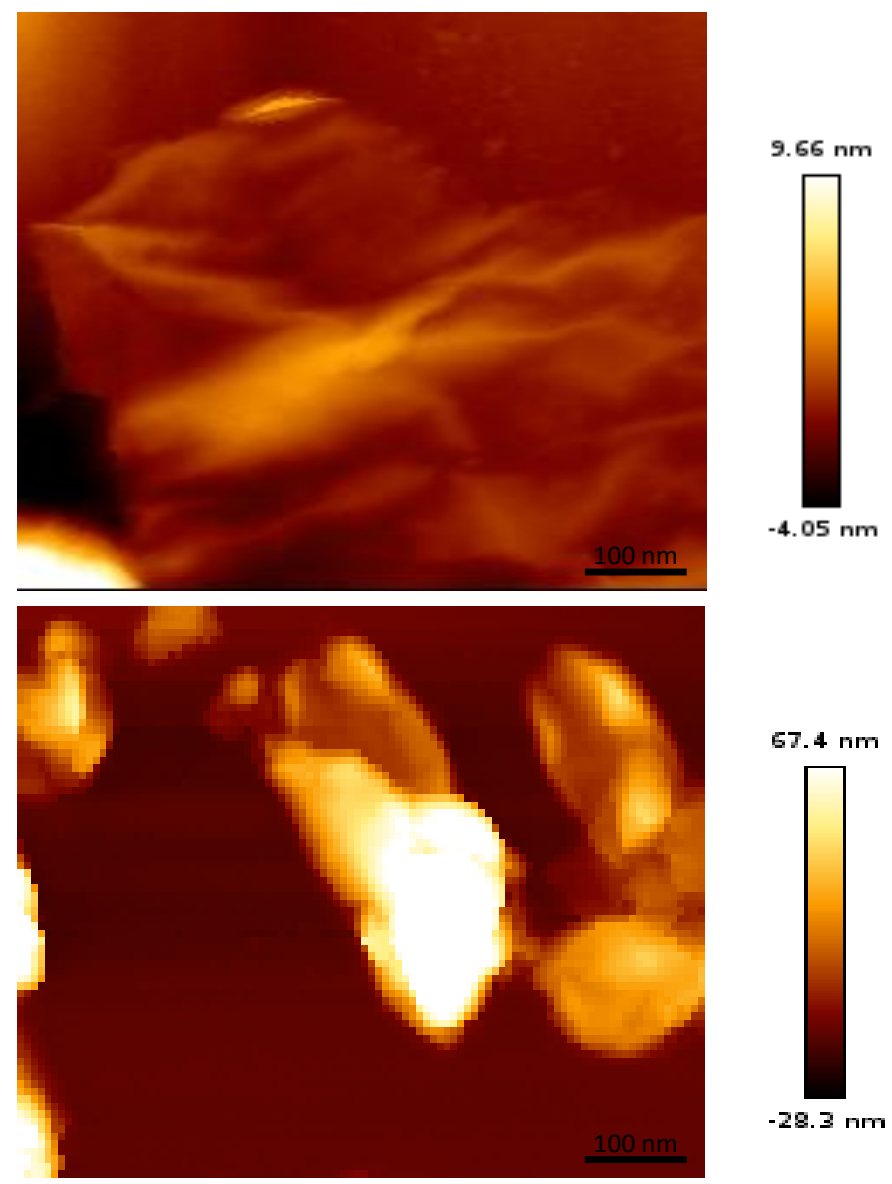

Figure 2. Representative AFM images of EGO 15 (top) and EGO 21 (bottom).

The image of EGO 14 clearly reveals the formation of very thin, homogeneous and good quality GO sheets, without traces from the pristine material. Very well exfoliated and wrinkled sheets can be detected, with a high degree of bending, in agreement with the presence of many oxygenated functional groups that can interact via $\mathrm{H}$-bonding. Thus, the application of $20 \mathrm{~V}$ for $60 \mathrm{~s}$ using $\mathrm{H}_{2} \mathrm{SO}_{4}$ as electrolyte is proven to be a very efficient exfoliation method. Regarding EGO 15, prepared under the same conditions yet using concentrated $\mathrm{H}_{2} \mathrm{SO}_{4}$ as electrolyte, the sheets were found to be slightly thicker, albeit their exfoliation level was also very good and the integrity of the graphene flakes was also preserved. Nonetheless, blocks of GO aggregates were detected for EGO 21, with flatter and smoother surfaces. The sheets could have broken during the second stage of the electrochemical process, resulting in sheets with smaller lateral sizes. Further, the presence of defects in the GO sheets can result in physical holes which generate poor waviness in the $\pi$-system. Overall, both techniques point that the EGOs with the lowest $\mathrm{C} / \mathrm{O}$ ratio, that is, the highest level of exfoliation (i.e., EGO 15) display the best exfoliation and the lowest flake thicknesses. 


\subsection{Specific Surface Area of the EGOs}

The specific surface area (SSA) for the reference GO and the synthesized EGOs was measured via the nitrogen adsorption-desorption technique. All the samples present SSA values in the range of 9 to $68 \mathrm{~m}^{2} \mathrm{~g}^{-1}$. The EGO 21 exhibits the lowest SSA, in agreement with the intense agglomeration of GO flakes as revealed by SEM and AFM. Conversely, EGO 14 exhibits the highest SSA, consistent with its improved exfoliation and well separated sheets. It has been reported that depending on the synthesis conditions such as temperature, electrolyte type and concentration, time, etc, SSA of GO can range from 4 up to $360 \mathrm{~m}^{2} \mathrm{~g}^{-1}$ [39].

In order to obtain more information about the parameters influencing SSA, this physical property was plotted against the inter-layer distance (also called d-spacing) corresponding to the (001) reflection obtained from the X-Ray diffractograms (Table 1) [37], and the results are plotted in Figure 3. A very good correlation is found between these two properties: as the d-spacing increases, the SSA raises. Thus, the largest interlayer spacing, close to $0.96 \mathrm{~nm}$, is obtained for EGO 14, which displays the highest SSA, while the smallest, about $0.85 \mathrm{~nm}$, are found for EGO 19 and 21. On the other hand, EGO 4 and 5, prepared under mild conditions, present in-between $d$ and SSA values. This means that the d-spacing grows with increasing the available surface area of the EGOs, suggesting that their structure becomes more regular and orientated. Analogous trend of increase in SSA with the interlayer distance has been reported for thermally reduced GO synthesized in the presence of CTAB surfactant [40], which intercalated between the nanomaterial layers. In our work, the intercalation of $\mathrm{SO}_{4}{ }^{2-}$ ions within the graphite layers during the first synthesis stage also results in significant interlayer spacing, and as it increases, SSA becomes larger.

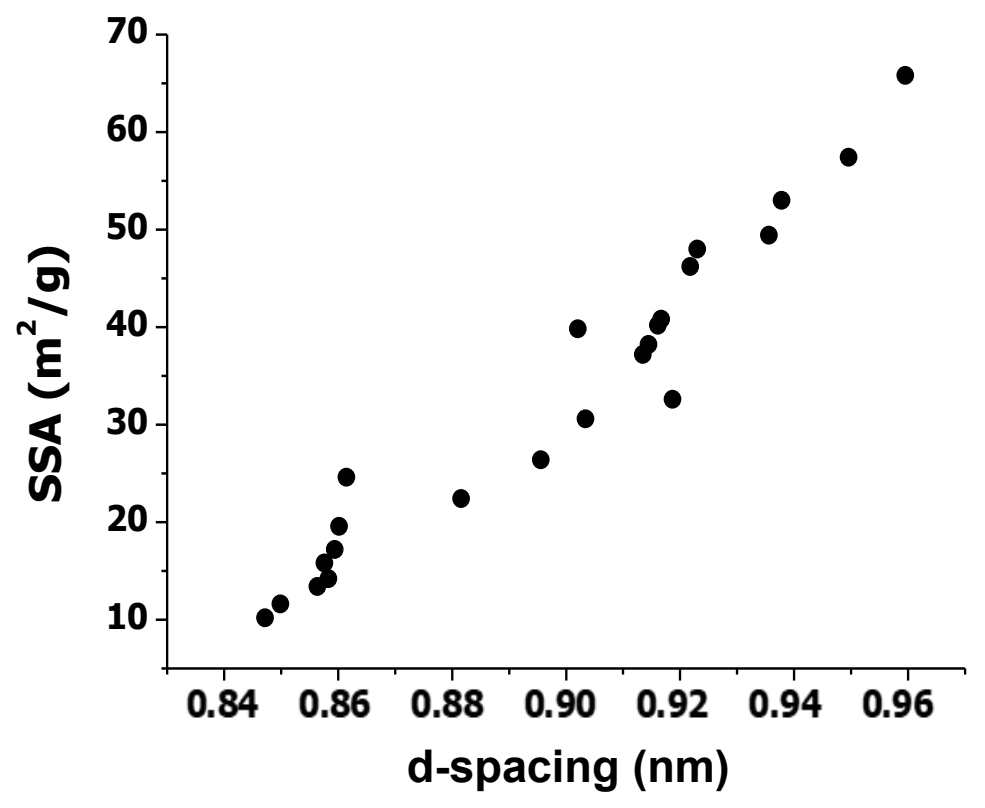

Figure 3. Specific surface area (SSA) vs. d-spacing for the synthesized EGOs.

To get insight about the influence of SSA on a bulk property, the electrical conductivity, the EGOs electrical resistance was measured and plotted against this parameter (Figure 4). In general, the electrical resistance decreases with increasing SSA. This behavior is expected considering that the increased surface area of the stacked GO sheets implies better degree of exfoliation, leading to a greater chance of forming percolated networks, hence enhanced electron mobility. This confirms that bulk properties are highly dependent on SSA, and consequently, on the sheet microstructure and also interlayer distance. 

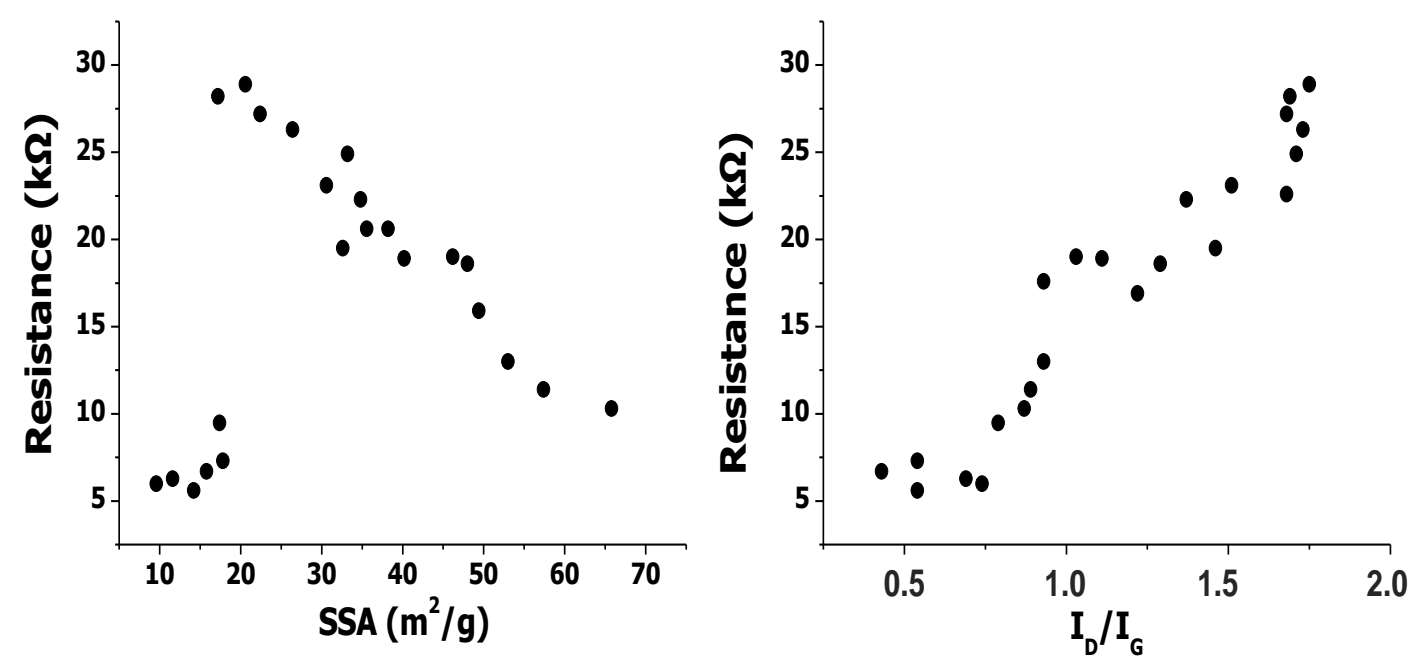

Figure 4. Electrical resistance vs. specific surface area SSA (left), and vs. ID/IG band intensity ratio obtained from the Raman spectra (right).

The electrical resistance was also plotted against the ID/IG ratio (the integrated intensity ratio of the $D$ peak and the G peak in the Raman spectrum, which is indicative of the quality of the GO layers and their defect content [41]. Thus, the $G$ band arises from the E2g vibrational mode found in graphite single crystal, and is characteristic of $\mathrm{sp}^{2}$ hybridization while the D band is related to defects, vacancies or lattice disorders due to the binding of oxygenated groups.

A direct relationship can be found between the electrical resistance and the ID/IG ratio, hence the defect content: as the ratio increases, the conductivity decreases. This behavior could be expected considering that graphene defects alter the length of the interatomic bond. They also change the type of the hybrid trajectories of the partial carbon atoms, and these changes affect the electrical properties. Point defects and single vacancy defects form an electron scattering center on the surface of graphene, resulting in decreased conductivity.

\section{Conclusions}

EGOs with various amounts of oxygenated groups have been synthesized by an electrochemical two-step process: intercalation followed by oxidation/exfoliation. For comparative purposes, a reference GO was prepared via a modified Hummers' method. The resulting GO samples have been characterized by different techniques in order to correlate their surface morphology with the macroscopic properties. SEM and AFM images reveal that the synthesis procedure used herein preserves the integrity of the graphene sheets, allowing to get large, homogenous and exfoliated GO layers. The macroscopic properties have been found to depend on the surface topography, interlayer spacing, defect content and specific surface area. The electrical resistance decreases with increasing specific surface area although rises with increasing the D/G band intensity ratio obtained from the Raman spectra, hence the defect content. The approach developed herein provides an effective approach to tailor the physical properties of nanomaterials incorporating GO for a variety of applications.

Author Contributions: C.S.-U and A.M.D.-P. performed the experiments and analyzed part of the data; A.M.D.P. designed the experiments, supervised the work and wrote the paper; M.P.S.A. and S.V-L. collaborated in the development of the experiments, in the analysis of the experimental data and the discussion of the results. All authors have read and agreed to the published version of the manuscript.

Acknowledgments: Financial support from the Spanish Ministry of Science, Innovation and Universities (MICIU) via Project PGC2018-093375-B-I00 is gratefully acknowledged.

Conflicts of Interest: The authors declare no conflict of interest. 


\section{References}

1. Yang, X.; Cheng, C.; Wang, Y.; Qiu, L.; Li, D. Liquid-mediated dense integration of graphene materials for compact capacitive energy storage. Science 2013, 341, 534-537, doi:10.1126/science.1239089.

2. Diez-Pascual, A.M.; Diez-Vicente, A.L. Poly(propylene fumarate)/polyethylene glycol-modified graphene oxide nanocomposites for tissue engineering. ACS Appl. Mater. Interfaces 2016, 8, 7902-17914, doi:10.1021/acsami.6b05635.

3. Diez-Pascual, A.M.; Chen, G. Selected Papers from the 1st International Online Conference on Nanomaterials. Nanomaterials 2019, 9, 1021, doi:10.3390/nano9071021.

4. Díez-Pascual, A.M.; Luceño Sánchez, J.A.; Peña Capilla, R.; García Díaz, P. Recent advances in graphene/polymer nanocomposites for applications in polymer solar cells. Polymers 2018, 10, 217, doi:10.3390/polym10020217.

5. Salavagione, H.; Díez-Pascual, A.M.; Lázaro, E.; Vera, S.; Gomez-Fatou, M. Chemical sensors based on polymer composites with carbon nanotubes and graphene: The role of the polymer. J. Mater. Chem. 2014, 2, 14289-14328, doi:10.1039/C4TA02159B.

6. Díez-Pascual, A.M.; Gómez-Fatou, M.A.; Ania, F.; Flores, A. Nanoindentation in Polymer Nanocomposites. Prog. Mater. Sci. 2015, 67, 1-94, doi:10.1016/j.pmatsci.2014.06.002.

7. Zhang, T.; Xue, Q.; Zhang, S.; Dong, M. Theoretical approaches to graphene and graphene-based materials. Nano Today 2012, 7, 180-200, doi:10.1016/j.nantod.2012.04.006.

8. Dreyer, D.R.; Park, S.; Bielawski, C.W.; Ruoff, R.S. The Chemistry of Graphene Oxide. Chem. Soc. Rev. 2010, 39, 228-240, doi:10.1039/B917103G.

9. Diez-Pascual, A.M.; Naffakh, M.; González-Domínguez, J.M.; Ansón, A.; Martinez Rubi, Y.; Martínez, M.T.; Simard, B.; Gómez, M.A. High performance PEEK/carbon nanotube composites compatibilized with polysulfones-I. Structure and thermal properties. Carbon 2010, 48, 3485-3499, doi:10.1016/j.carbon.2010.05.046.

10. Diez-Pascual, A.M.; Valles, C.; Mateos, R.; Vera-López, S.; Kinloch, I.A.; San Andrés M.P. Influence of surfactants of different nature and chain length on the morphology, thermal stability and sheet resistance of graphene. Soft Matter 2018, 14, 6013-6023, doi:10.1039/C8SM01017J.

11. Luceño Sánchez, J.A.; Peña Capilla, R.; Díez-Pascual, A.M. High-Performance PEDOT:PSS/Hexamethylene Diisocyanate-Functionalized Graphene Oxide Nanocomposites: Preparation and Properties. Polymers 2018, 10, 1169, doi:10.3390/polym10101169.

12. Diez-Pascual, A.M.; Diez-Vicente, A.L. Multifunctional poly(glycolic acid-co-propylene fumarate) electrospun fibers reinforced with graphene oxide and hydroxyapatite nanorods. J. Mater. Chem. B 2017, 5 , 4084-4096, doi:10.1039/C7TB00497D.

13. Valles, C.; Beckert, F.; Burk, L.; Mülhaupt, R.; Young, R.J.; Kinloch, I.A. Effect of the C/O ratio in graphene oxide materials on the reinforcement of epoxy-based nanocomposites. J. Polym. Sci. Part B Polym. Phys. 2016, 54, 281-291, doi:10.1002/polb.23925.

14. Luceño-Sanchez, J.A.; Diez-Pascual, A.M. Grafting of Polypyrrole-3-carboxylic Acid to the Surface of Hexamethylene Diisocyanate-Functionalized Graphene Oxide. Nanomaterials 2019, 9, 1095, doi:10.3390/nano9081095.

15. Luceño-Sánchez, J.A.; Maties, G.; Gonzalez-Arellano, C.; Diez-Pascual, A.M. Synthesis and Characterization of Graphene Oxide Derivatives via Functionalization Reaction with Hexamethylene Diisocyanate. Nanomaterials 2018, 8, 870, doi:10.3390/nano8110870.

16. Fan, W.; Zhang, C.; Tjiu, W.W.; Liu, T. Fabrication of electrically conductive graphene/polystyrene composites via a combination of latex and layer-by-layer assembly approaches. J. Mater. Res. 2013, 28, 611619, doi:10.1557/jmr.2012.437.

17. Luceño Sanchez, J.A.; Diez-Pascual, A.M.; Peña Capilla, R.; García Diaz, P. The Effect of Hexamethylene Diisocyanate-Modified Graphene Oxide as a Nanofiller Material on the Properties of Conductive Polyaniline. Polymers 2019, 11, 1032, doi:10.3390/polym11061032.

18. Pruna, A.; Pullini, D.; Busquets, D. Influence of synthesis conditions on properties of green-reduced graphene oxide. J. Nanoparticle Res. 2013, 15, 1-11, doi:10.1007/s11051-013-1605-6.

19. Bhuyan, M.S.A.; Uddin, M.N.; Islam, M.M.; Bipasha, F.A.; Hossain, S.S. Synthesis of graphene. Int. Nano Lett. 2016, 6, 65-83, doi:10.1007/s40089-015-0176-1.

20. Gupta, V.; Sharma, N.; Singh, U.; Arif, M.; Singh, A. Higher oxidation level in graphene oxide. Optik 2017, 143, 115-124, doi:10.1016/j.ijleo.2017.05.100. 
21. Liu, L.; Zhang, F.; Zhao, J.; Liu, F. Mechanical properties of graphene oxides. Nanoscale 2012, 4, 5910-5916, doi:10.1039/C2NR31164J.

22. Lyn, F.H.; Peng, T.C.; Ruzniza, M.Z.; Hanani, Z.A.N. Effect of oxidation degrees of graphene oxide (GO) on the structure and physical properties of chitosan/GO composite films. Food Packag. Shelf 2019, 21, 100373, doi:10.1016/j.fpsl.2019.100373.

23. Scaffaro, R.; Maio, A. Influence of oxidation level of graphene oxide on the mechanical performance and photo-oxidation resistance of a polyamide 6. Polymers 2019, 11, 857, doi:10.3390/polym11050857.

24. Bandara, N.; Esparza, Y.; Wu, J. Graphite oxide improves adhesion and water resistance of canola proteingraphite oxide hybrid adhesive. Sci. Rep. 2017, 7, 11538, doi:10.1038/s41598-017-11966-8.

25. Morimoto, N.; Kubo, T.; Nishina, Y. Tailoring the oxygen content of graphite and reduced graphene oxide for specific applications. Sci. Rep. 2016, 6, 21715, doi:10.1038/srep21715.

26. Park, S.; Ruoff, R.S. Chemical methods for the production of graphenes. Nat. Nanotechnol. 2009, 4, 217-224, doi:10.1038/nnano.2009.58.

27. Brodie, DC. On the Atomic Weight of Graphite. Trans. R. Soc. 1859, 149, 249-259, doi:10.1098/rstl.1859.0013.

28. Hummers, W.S.; Offeman, R.E. Preparation of graphitic oxide. J. Am. Chem. Soc. 1958, 80, 1339-1339, doi:10.1021/ja01539a017.

29. Chen, J.; Yao, B.; Li, C.; Shi, G. An improved Hummers method for eco-friendly synthesis of graphene oxide. Carbon 2013, 64, 225-229, doi:10.1016/j.carbon.2013.07.055.

30. Yang, S.; Lohe, M.R.; Muellen, K.; Feng, X. New-generation graphene from electrochemical approaches: Production and applications. Adv. Mater. 2016, 28, 6213-6221, doi:10.1002/adma.201505326.

31. Yang, S.; Brüller, S.; Wu, Z.S.; Liu, Z.; Parvez, K.; Dong, R.; Richard, F.; Samorì, P.; Feng, X.; Müllen, K. Organic radical-assisted electrochemical exfoliation for the scalable production of high-quality graphene. J. Am. Chem. Soc. 2015, 137, 13927-13932, doi:10.1021/jacs.5b09000.

32. Liu, J.; Yang, H.; Zhen, S.G.; Poh, C.K.; Chaurasia, A.; Luo, J.; Wu, X.; Yeow, E.K.L.; Sahoo, N.G.; Lin, J.; et al. A green approach to the synthesis of high-quality graphene oxide flakes via electrochemical exfoliation of pencil core. RSC Adv. 2013, 3, 11745-11750, doi:10.1039/C3RA41366G.

33. Gurzęda, B.; Florczak, P.; Kempiński, M.; Peplińska, B.; Krawczyk, P.; Jurga, S. Synthesis of graphite oxide by electrochemical oxidation in aqueous perchloric acid. Carbon 2016, 100, 540-545, doi:10.1016/j.carbon.2016.01.044.

34. Parvez, K.; Rincón, R.A.; Weber, N.E.; Chaa, K.C.; Venkataraman, S.S. One-step electrochemical synthesis of nitrogen and sulfur co-doped, high-quality graphene oxide. Chem. Commun. 2016, 52, 5714-5717, doi:10.1039/C6CC01250G.

35. Ustavytska, O.; Kurys, Y.; Koshechko, V.; Pokhodenko, V. One-step electrochemical preparation of multilayer graphene functionalized with nitrogen. Nanoscale Res. Lett. 2017, 12, 175, doi:10.1186/s11671-017$1957-4$

36. Kang, F.; Leng, Y.; Zhang, T.Y. Influences of H2O2 on Synthesis of H2SO4-GICs. J. Phys. Chem. Solids 1996, 57, 889-892, doi:10.1016/0022-3697(95)00368-1.

37. Diez-Pascual, A.M.; Sainz-Urruela, C.; Vallés, C.; Vera-López, S.; San Andrés, M.P. Tailorable Synthesis of Highly Oxidized Graphene Oxides via an Environmentally-Friendly Electrochemical Process. Nanomaterials 2020, 10, 239, doi:10.3390/nano10020239.

38. Gao, W.; Alemany, L.B.; Ci, L.; Ajayan, P.M. New insights into the structure and reduction of graphite oxide, Nat. Chem. 2009, 1, 403-408, doi:10.1038/nchem.281.

39. Alazmi, A.; Tall, O.E.; Rasul, S.; Hedhili, M.N.; Patole, S.P.; Costa, P.M.F.J. A process to enhance the specific surface area and capacitance of hydrothermally reduced graphene oxide. Nanoscale 2016, 8, 17782-17787, doi:10.1039/C6NR04426C.

40. Lin, J.H. The Influence of the Interlayer Distance on the Performance of Thermally Reduced Graphene Oxide Supercapacitors. Materials 2018, 8, 263, doi:10.3390/ma11020263.

41. Tuinstra, F.; Koenig, J.L. Raman Spectrum of Graphite. J. Chem. Phys. 1970, 53, 1126, doi:10.1063/1.1674108.

Publisher's Note: MDPI stays neutral with regard to jurisdictional claims in published maps and institutional affiliations.

(C) 2020 by the authors. Submitted for possible open access publication under the terms and conditions of the Creative Commons Attribution (CC BY) license (http://creativecommons.org/licenses/by/4.0/). 